\title{
STEREOSCOPIC VISION IN RELATION TO PHOTO-INTERPRETATION*
}

BY

\author{
ALAN A. MORGAN \\ Dagenham, Essex
}

SINCE the intelligence reports issued during the war depended partly on the proper interpretation of operational photographs, this subject acquired considerable importance.

The final report on an operational photograph depends on several qualities of the interpreter, particularly his specialized knowledge of the subject and his stereoscopic sense.

Stereoscopic vision or depth perception is a distinct property of the cones of the fovea and is dependent on the stimulation of disparate points in the retinae. Generally, one utilizes associated factors (e.g. size, shadows, etc.), in determining the position of objects in space, so that true stereoscopic sense is not really developed in the average person.

An attempt has been made to determine the value of stereoscopic training in photo-interpretation and to ascertain the effect of suppression changes and refractive errors on stereoscopic vision.

\section{Method}

As many personnel as possible were graded stereoscopically and then divided into two groups. One group were stereoscopically trained and the others acted as controls. The grading and training were carried out on Holmes' stereoscopes with the rotating stereograms of Livingston (1939). The initial grading was done on Charts 1 and 5, a note of any abnormality in convergence or simultaneous perception being made at the time. The training was carried out progressively on Charts $4,6,10,9$, and 7 . At the end of the training period, the trainees were examined with their controls (as far as possible the controls had an original grading similar to the trainees). The final test was carried out on Charts 5 and 8 of the series and also on an operational photograph. Chart 5, which is a difficult one, and on which the initial grading was done, enables one to see what improvement the training has had on their stereoscopic sense. Chart 8 had not been seen previously by either the trained or untrained personnel. The operational photograph chosen for the final test had particular stereoscopic properties, so that, if only associated factors were utilized in depth perception, wrong conclusions might be drawn, and the exercise of true stereoscopic vision was needed for its accurate interpretation.

The trained personnel went through a graduated course of twenty sessions on Charts 4, 6, 10, 9, and 7-an average session lasting 10-15 minutes. 


\section{$\dot{0}+\mid$}

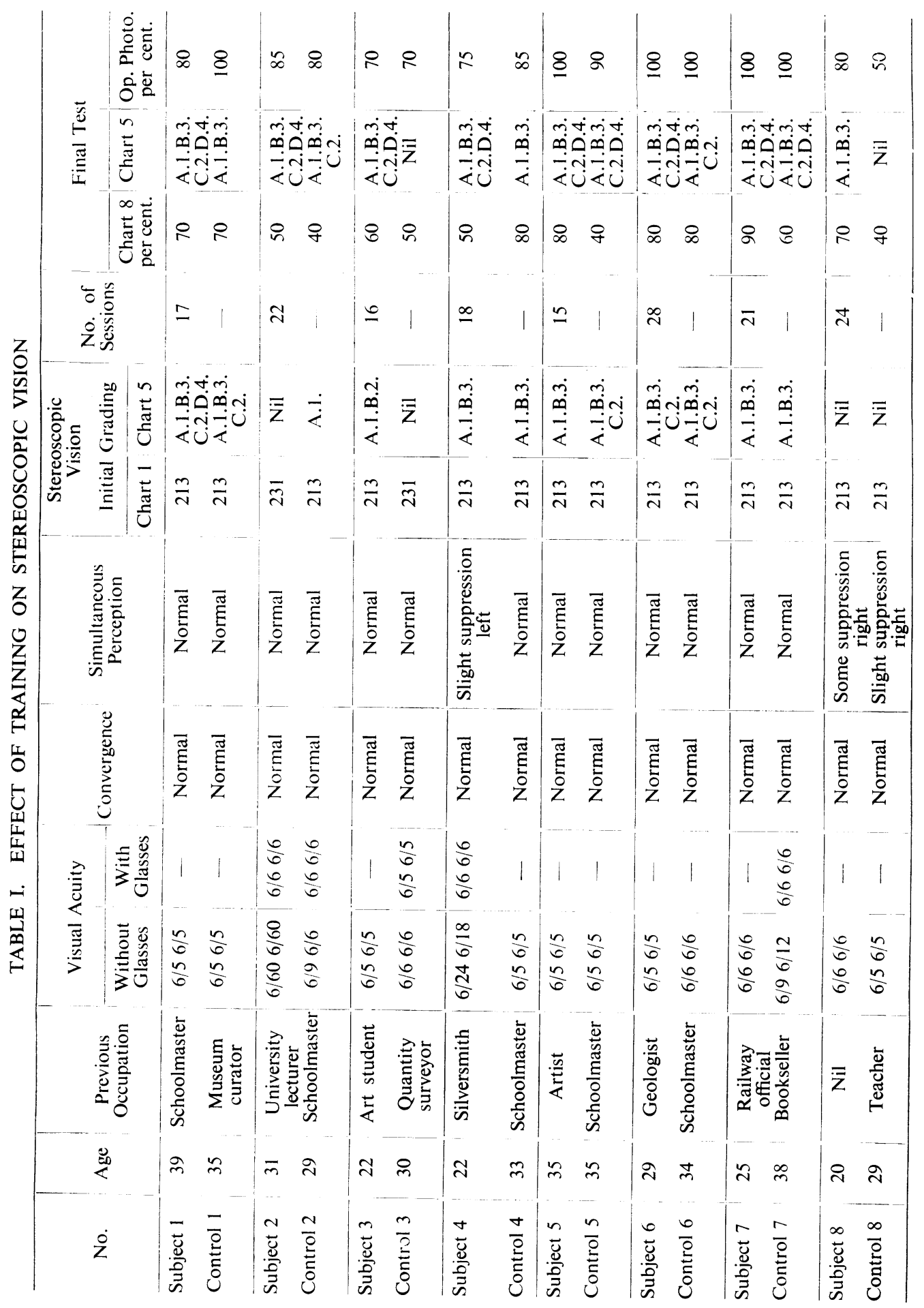


Owing to shift work and other factors it actually took 3 or 4 months to complete a course, and throughout this time both the trainees and their controls were actively engaged in photo-interpretation. To enable the results to be understood, it may be pointed out that the correct answers to Charts 1 and 5 are 213 and A.1.B.3.C.2.D.4. respectively.

Effect of Training (Table I).

\section{Results}

The number of personnel concerned is too small to draw very definite conclusions, but the Table suggests the following points:

(a) All the trained personnel show a definite improvement on Chart 5 after their course of training.

(b) The majority of untrained personnel show little or no improvement on Chart 5.

(c) On the operational photograph there seems very little to choose between trained and untrained personnel, but, on the whole, the trained did a little better.

These points indicate that a definite improvement in stereoscopic vision results from steresocopic training. Furthermore, it appears that personnel engaged on stereoscopic work have their stereoscopic vision automatically improved without undergoing any special stereoscopic training, although this happens more slowly than with systematic graduated training. It is felt that even better results could be produced in the trained candidates if the training sessions had been on consecutive days, and that a greater contrast would then have been evident between the trained and untrained personnel on the operational photograph as well, as after only 3 weeks (the time needed for a concentrated course of training) the untrained personnel would not have been carrying out their daily work long enough to make any appreciable improvement in their stereoscopic vision.

Effect of Suppression Changes (Table II).

Section A contains subjects with complete or nearly complete suppression

TABLE II

\begin{tabular}{|c|c|c|c|c|c|c|c|c|c|c|}
\hline \multirow[b]{2}{*}{ Section } & \multirow[b]{2}{*}{ No. } & \multirow[b]{2}{*}{ Age } & \multirow[b]{2}{*}{ Convergence } & \multirow[b]{2}{*}{ Simultaneous Perception } & \multicolumn{2}{|c|}{ Stereoscopic Vision } & \multicolumn{4}{|c|}{ After a Course of Training } \\
\hline & & & & & Chart 1 & Chart 5 & $\underset{1}{\text { Chart }}$ & $\underset{5}{\text { Chart }}$ & $\begin{array}{c}\text { Chart } \\
8 \\
\text { per } \\
\text { cent. }\end{array}$ & $\begin{array}{l}\text { Op. } \\
\text { Photo } \\
\text { per } \\
\text { cent. }\end{array}$ \\
\hline A & $\begin{array}{l}1 \\
2 \\
3\end{array}$ & $\begin{array}{l}55 \\
38 \\
19\end{array}$ & $\begin{array}{l}\text { Normal } \\
\text { Normal } \\
\text { Normal }\end{array}$ & $\begin{array}{l}\text { Complete suppression left } \\
\text { Complete suppression left } \\
\text { Considerable suppression } \\
\text { right }\end{array}$ & $\begin{array}{l}123 \\
\mathrm{Nil} \\
123\end{array}$ & $\begin{array}{l}\mathrm{Nil} \\
\mathrm{Nil} \\
\mathrm{Nil}\end{array}$ & 123 & Nil & 10 & 0 \\
\hline \multirow{3}{*}{ B } & $\begin{array}{l}4 \\
5\end{array}$ & $\begin{array}{l}37 \\
35\end{array}$ & $\begin{array}{l}\text { Normal } \\
\text { Normal }\end{array}$ & $\begin{array}{c}\text { Some suppression right } \\
\text { Normal }\end{array}$ & $\begin{array}{l}213 \\
213\end{array}$ & $\begin{array}{l}\text { Nil } \\
\text { A.1.B.3.C.2. }\end{array}$ & & & & \\
\hline & $\begin{array}{l}6 \\
7\end{array}$ & $\begin{array}{l}40 \\
34\end{array}$ & $\begin{array}{l}\text { Normal } \\
\text { Normal }\end{array}$ & $\begin{array}{c}\text { Some suppression right } \\
\text { Normal }\end{array}$ & $\begin{array}{l}123 \\
213\end{array}$ & $\begin{array}{l}\text { Nil } \\
\text { A.1.B.3.C.2. } \\
\text { D.4. }\end{array}$ & & & & \\
\hline & $\begin{array}{l}8 \\
9\end{array}$ & $\begin{array}{l}36 \\
29\end{array}$ & $\begin{array}{l}\text { Normal } \\
\text { Normal }\end{array}$ & Some suppression right & $\begin{array}{l}231 \\
213\end{array}$ & $\begin{array}{l}\text { A.1. } \\
\text { A.1.B.3.C.2. }\end{array}$ & & & & \\
\hline $\mathrm{C}$ & $\begin{array}{l}10 \\
11\end{array}$ & $\begin{array}{l}20 \\
29\end{array}$ & $\begin{array}{l}\text { Normal } \\
\text { Normal }\end{array}$ & $\begin{array}{l}\text { Some suppression left } \\
\text { Some suppression right }\end{array}$ & $\begin{array}{l}213 \\
213\end{array}$ & $\stackrel{\text { Nil }}{\text { A.1.B.3.C.2. }}$ & $\begin{array}{l}213 \\
213\end{array}$ & $\begin{array}{l}\text { A.1.B.3 } \\
\text { A.1.B.3 } \\
\text { C.2.D. } 4\end{array}$ & & \\
\hline
\end{tabular}


of one eye and it is seen that they exhibit no stereoscopic sense. Subject No. 1 was given a trial of 7 days' continuous training, but not the slightest improvement in his suppression or stereoscopic sense could be detected.

Section B compares those with partial suppression and those with no suppression. It illustrates that the cases of partial suppression have worse stereoscopic vision than the others.

Section $\mathrm{C}$ shows the effect of exercises on those with partial suppression and suggests that they may be amenable to training. Incidentally, these two candidates required the most sessions to complete their training (28 and 24 respectively), exercises to overcome suppression being necessary before stereoscopic training could be begun.

Generally, therefore, those with complete or nearly complete suppression are unsuitable for photo-interpretation. Those that show partial suppression should only be accepted for the work as second choice after those who show no suppression, and then only after a week's trial to find out whether they are amenable to training. If they are, they should then undergo a complete course of stereoscopic training.

Effect of Refractive Errors (Tables III, IV, V, and VI).

Table III shows subjects with refractive errors but good stereoscopic vision, and Table IV those with refractive errors but poor stereoscopic vision. The fact that Subject No. 6 developed satisfactory stereoscopic vision after training suggests that refractive error is no handicap to successful stereoscopic training.

TABLE III

\begin{tabular}{|c|c|c|c|c|c|c|}
\hline \multirow{3}{*}{ No. } & \multirow{3}{*}{ Age } & \multirow{3}{*}{ Convergence } & \multirow{3}{*}{$\begin{array}{c}\text { Simultaneous } \\
\text { Perception }\end{array}$} & \multirow{2}{*}{\multicolumn{2}{|c|}{$\frac{\text { Stereoscopic Vision }}{\text { Initial Grading }}$}} & \multirow{3}{*}{ Visual Defects } \\
\hline & & & & & & \\
\hline & & & & Chart 1 & Chart 5 & \\
\hline 1 & 21 & Normal & Normal & 213 & A.1.B.3.C.2.D.4. & Mild hypermetropic as- \\
\hline 2 & 34 & Normal & Normal & 213 & A.1.B.3.C.2. & Mild hypermetropic as- \\
\hline 3 & 20 & Normal & Normal & 213 & A.1.B.3.C.2. & $\begin{array}{l}\text { tigmantism } \\
\text { Hypermetropic astig- }\end{array}$ \\
\hline 4 & 36 & Normal & Normal & 213 & A.1.B.3.C.2. & $\begin{array}{l}\text { matism } \\
\text { Myopic astigmatism }\end{array}$ \\
\hline $\begin{array}{l}5 \\
6\end{array}$ & $\begin{array}{l}35 \\
23\end{array}$ & $\begin{array}{l}\text { Normal } \\
\text { Normal }\end{array}$ & $\begin{array}{l}\text { Normal } \\
\text { Normal }\end{array}$ & $\begin{array}{l}213 \\
213\end{array}$ & $\begin{array}{l}\text { A.1.B.3.C.2. } \\
\text { A.1.B.3.C.2.D.4. }\end{array}$ & $\begin{array}{l}\text { especially right eye } \\
\text { Moderate myopia } \\
\text { Hypermetropic astig- }\end{array}$ \\
\hline 7 & 25 & Normal & Normal & 213 & A.1.B.2.C.3.D.4. & $\begin{array}{l}\text { matism } \\
\text { Hypermetropic astig- }\end{array}$ \\
\hline 8 & 32 & Normal & Normal & 213 & A.1.B.3.C.2.D.4. & $\begin{array}{l}\text { matism } \\
\text { Severe myopia. Visual } \\
\text { Acuity less than } 1 / 60 \\
\text { in each eye }\end{array}$ \\
\hline
\end{tabular}


TABLE IV

\begin{tabular}{|c|c|c|c|c|c|c|c|c|c|}
\hline \multirow{3}{*}{ No. } & \multirow{3}{*}{ Age } & \multirow{3}{*}{ Convergence } & \multirow{3}{*}{$\begin{array}{c}\text { Simultaneous } \\
\text { Perception }\end{array}$} & \multirow{2}{*}{\multicolumn{2}{|c|}{$\begin{array}{c}\text { Stereoscopic Vision } \\
\text { Initial Grading }\end{array}$}} & \multicolumn{3}{|c|}{ Final Grading } & \multirow{3}{*}{ Visual Defects } \\
\hline & & & & & & \multirow{2}{*}{$\begin{array}{l}\text { Chart } 8 \\
\text { per cent. }\end{array}$} & \multirow{2}{*}{ Chart 5} & \multirow{2}{*}{$\begin{array}{c}\text { Op. } \\
\text { Photo } \\
\text { per cent. }\end{array}$} & \\
\hline & & & & Chart 1 & Chart 5 & & & & \\
\hline $\begin{array}{l}1 \\
2 \\
3 \\
4\end{array}$ & $\begin{array}{l}34 \\
20 \\
26 \\
30\end{array}$ & $\begin{array}{l}\text { Normal } \\
\text { Normal } \\
\text { Normal } \\
\text { Normal }\end{array}$ & $\begin{array}{l}\text { Normal } \\
\text { Normal } \\
\text { Normal } \\
\text { Normal }\end{array}$ & $\begin{array}{l}213 \\
213 \\
213 \\
231\end{array}$ & $\begin{array}{l}\text { Nil } \\
\text { Nil } \\
\text { Nil } \\
\text { Nil }\end{array}$ & & & & $\begin{array}{l}\text { Myopic astigmatism } \\
\text { Myopia }+++ \\
\text { Moderate myopia } \\
\text { Hypermetropic astig- }\end{array}$ \\
\hline 5 & 35 & Normal & Normal & 213 & Nil & & & & Hypermetropic astig- \\
\hline 6 & 31 & Normal & Normal & 231 & Nil & 50 & $\begin{array}{l}\text { A.1.B.3. } \\
\text { C.2.D.4. }\end{array}$ & 85 & Myopia +++ \\
\hline
\end{tabular}

Table V shows subjects with normal visual acuity and good stereoscopic vision, and Table VI those with normal visual acuity but poor stereoscopic vision.

TABLE V

\begin{tabular}{|c|c|c|c|c|c|c|}
\hline \multirow{3}{*}{ No. } & \multirow{3}{*}{ Age } & \multirow{3}{*}{ Convergence } & \multirow{3}{*}{$\begin{array}{l}\text { Simultaneous } \\
\text { Perception }\end{array}$} & \multicolumn{2}{|c|}{ Stereoscopic Vision } & \multirow{3}{*}{ Visual Defects } \\
\hline & & & & \multicolumn{2}{|c|}{ Initial Grading } & \\
\hline & & & & Chart 1 & Chart 5 & \\
\hline $\begin{array}{l}1 \\
2 \\
3 \\
4 \\
5 \\
6 \\
7 \\
8\end{array}$ & $\begin{array}{l}20 \\
28 \\
31 \\
48 \\
24 \\
30 \\
27 \\
18\end{array}$ & $\begin{array}{l}\text { Normal } \\
\text { Normal } \\
\text { Normal } \\
\text { Normal } \\
\text { Normal } \\
\text { Normal } \\
\text { Normal } \\
\text { Normal }\end{array}$ & $\begin{array}{l}\text { Normal } \\
\text { Normal } \\
\text { Normal } \\
\text { Normal } \\
\text { Normal } \\
\text { Normal } \\
\text { Normal } \\
\text { Normal }\end{array}$ & $\begin{array}{l}213 \\
213 \\
213 \\
213 \\
213 \\
213 \\
213 \\
213\end{array}$ & $\begin{array}{l}\text { A.1.B.3.C.2.D.4. } \\
\text { A.1.B.3.C.2. } \\
\text { A.1.B.3.C.2.D.4 } \\
\text { A.1.B.3.C.2.D.4 } \\
\text { A.1.B.3.C.2.D.44 } \\
\text { A.1.B.3.C.2.D.44 } \\
\text { A.1.B.3.C.2.D.4. } \\
\text { A.1.B.3.C.2.D.4 }\end{array}$ & $\begin{array}{l}\text { Nil } \\
\text { Nil } \\
\text { Nil } \\
\text { Nil } \\
\text { Nil } \\
\text { Nil } \\
\text { Nil } \\
\text { Nil }\end{array}$ \\
\hline
\end{tabular}

TABLE VI

\begin{tabular}{|c|c|c|c|c|c|c|}
\hline \multirow{3}{*}{ No. } & \multirow{3}{*}{ Age } & \multirow{3}{*}{ Convergence } & \multirow{3}{*}{$\begin{array}{c}\text { Simultaneous } \\
\text { Perception }\end{array}$} & \multicolumn{2}{|c|}{ Stereoscopic Vision } & \multirow{3}{*}{ Visual Defects } \\
\hline & & & & \multicolumn{2}{|c|}{ Initial Grading } & \\
\hline & & & & Chart 1 & Chart 5 & \\
\hline $\begin{array}{r}1 \\
2 \\
3 \\
4 \\
5 \\
6 \\
7 \\
8 \\
9 \\
10 \\
11\end{array}$ & $\begin{array}{l}26 \\
33 \\
33 \\
24 \\
34 \\
24 \\
22 \\
21 \\
41 \\
29 \\
22\end{array}$ & $\begin{array}{l}\text { Normal } \\
\text { Normal } \\
\text { Normal } \\
\text { Normal } \\
\text { Normal } \\
\text { Normal } \\
\text { Normal } \\
\text { Normal } \\
\text { Normal } \\
\text { Normal } \\
\text { Normal }\end{array}$ & $\begin{array}{l}\text { Normal } \\
\text { Normal } \\
\text { Normal } \\
\text { Normal } \\
\text { Normal } \\
\text { Normal } \\
\text { Normal } \\
\text { Normal } \\
\text { Normal } \\
\text { Normal } \\
\text { Normal }\end{array}$ & $\begin{array}{l}213 \\
213 \\
213 \\
213 \\
231 \\
213 \\
231 \\
213 \\
213 \\
213 \\
213\end{array}$ & $\begin{array}{l}\text { A.1. } \\
\text { Nil } \\
\text { Nil } \\
\text { Nil } \\
\text { A.1.B.3. } \\
\text { A.1. } \\
\text { A.1. } \\
\text { Nil } \\
\text { A.1. } \\
\text { Nil } \\
\text { Nil }\end{array}$ & $\begin{array}{l}\text { Nil } \\
\text { Nil } \\
\text { Nil } \\
\text { Nil } \\
\text { Nil } \\
\text { Nil } \\
\text { Nil } \\
\text { Nil } \\
\text { Nil } \\
\text { Nil } \\
\text { Nil }\end{array}$ \\
\hline
\end{tabular}


These results show that there is no correlation between refractive errors and stereoscopic vision.

\section{Conclusions}

It has been noticed that it is not uncommon for personnel to complain of ocular symptoms such as tiredness of the eyes and headaches after beginning to use the stereoscope in photo-interpretation. This is merely due to ocular fatigue, probably resulting from the fact that the ideal way of looking at a photograph is to dissociate accommodation from convergence. This, plus the bending over whilst looking at photographs with its associated increased intra-ocular tension and impaired intra-cranial venous drainage is probably responsible for the symptoms. They usually wear off in a week or two.

Stereoscopic work per se has no evil effects on the eyes, and there is no reason at all why any refractive errors already present should be increased as a result of the stereoscopic work, providing it is done under proper conditions, especially with adequate lighting and good arrangements of desks and chairs. It is recommended that there should be a uniform illumination of the whole operational room, plus a slightly greater illumination of the photograph under observation. There should not be much contrast between the illumination on the photograph and the surroundings, as adaptation changes would then come into play and might produce further symptoms. It is also recommended that the ledge of the desk on which the operational photograph rests for stereoscopic examination should be far more vertical than that in general use.

As regards standards for recruits undertaking photo-interpretation, it seems that suppression changes are far more important than visual acuity, and since the type of person often found suitable for the work is of the academic class, it is not uncommon to find refractive errors present.

The following standards are recommended:

1. Visual Acuity. $-6 / 60$ or better in each eye, correctable to $6 / 6$.

2. Convergence. -4 " or better.

3. No cases of complete suppression should be accepted.

4. Cases with partial suppression should only be accepted with the aforementioned proviso (Table II).

As regards those who may be particularly well qualified for photo-interpretation, the standard may be relaxed in regard to visual acuity after testing their stereoscopic vision, but not in regard to suppression changes.

It is also recommended that all personnel, irrespective of their initial grading, should have a complete course of stereoscopic training with daily exercises.

I wish to thank Air Marshal Sir P. C. Livingston, K.B.E., C.B., A.F.C., formerly_Consultant in Ophthalmology, Royal Air Force, for his help and interest in this work.

\section{REFERENCE}

Luvingston, P. C. (1939). British Journal of Ophthalmology, 23, 540. 